\title{
Gender Wise Association between Dental Malocclusion Classes and ABO Blood Group System
}

Bushra Tariq*, Kainat Habib, Sidra Riaz and Muhammad llyas

Department of Dental, University of Health Sciences, Lahore, Punjab, Pakistan

*Corresponding author: Bushra Tariq, Department of Dental, University of Health Sciences, Lahore, Punjab, Pakistan, Tel: +923041606234; E-mail: bushratariq427@gmail.com

Received date: January 11, 2019; Accepted date: February 04, 2019; Published date: February 08, 2019

Copyright: (c) 2019 Tariq B, et al. This is an open-access article distributed under the terms of the Creative Commons Attribution License, which permits unrestricted use, distribution, and reproduction in any medium, provided the original author and source are credited.

\begin{abstract}
Background: Malocclusion shares the third highest prevalence among oral pathologies, second to tooth decay and periodontal diseases.

Objectives: In general, many studies have been conducted to find out the association between blood groups and oral and dental pathologies, for instance, some have succeeded; others have not due to the geographic diversity. So the study aimed to explore the relationship of $A B O$ blood groups with classes of malocclusion and relative prevalence of these characters in males and females of a defined population.

Method: The current study was performed on 500 individuals with an age range of 15 to 45 years, along with permanent dentition only, reporting to the department of orthodontics of Punjab dental hospital, Lahore. Complete details about the classes of malocclusion and blood group of the subjects were noted in accordance with the ABO blood group system. The sample was divided into three groups, i.e., Class I, Class II, and Class III. Data were analyzed statistically by using SPSS 21.0. Pearson Chi-square was used as statistical analysis to get the association of classes of malocclusion with blood group type.
\end{abstract}

Result: A significant difference between the prevalence of classes of dental malocclusion in relation to blood group types was found among both genders.

Conclusion: In conclusion, there was gender wise association difference between the classes of dental malocclusion and blood group types. The study also covered the gender wise prevalence of dental malocclusion in all the four blood groups which are $A, B, A B$ and $O$. The new findings will be helpful for clinicians with regards to preventive orthodontics. The research findings are a good and informative addition to the present literature as well as provides a ground for further research studies in the same area.

Keywords: ABO blood group system; Dental malocclusion classes; Gender wise prevalence; Oral pathologies; Preventive orthodontics

\section{Introduction}

Malocclusion is the abnormal relationship between the teeth of two dental arches when they approximate each other as the jaws close, thus it is considered as an unacceptable deviation from the ideal occlusion [1]. Malocclusion shares the third highest prevalence among oral pathologies, second to tooth decay and periodontal diseases.

Malocclusion is a multifactorial trait. Graber stated the etiological factors of malocclusion as general factors, such as hereditary and genetic defects, environmental anomalies, dietary problems, abnormal habits, and functional aberrations and trauma or local factors, such as anomalies in tooth numbers, size, shape, premature loss and prolonged retention of primary teeth. The relative contribution of genetics and the environment to the etiology of malocclusion has been a matter of controversy throughout the $20^{\text {th }}$ century, yet there is irrefutable evidence for significant genetic influence on malocclusion [2]. In the late $20^{\text {th }}$ century, Dobzhansky, et al. [3] stated three possible types of transmission of malocclusions from the standpoint of genetics as:
- Repetitive: the recurrence of a single dentofacial deviation within the immediate family

- Discontinuous: the recurrence of a tendency for a malocclusal trait to reappear in a family background over several generations

- Variable: occurrence of different but related malocclusions within several generations of the same family

Epidemiological evaluations can reveal the association between malocclusion and some other genetic traits. After the discovery of a blood group system "one of the most important human genetic trait" many attempts were made to find the association between blood group antigens and different oral conditions. The $\mathrm{ABO}$ blood group system was discovered by Karl Landsteiner in 1901, who found three different blood types A, B and O [4]. In 1902, Decastello and Sturli discovered the $4^{\text {th }}$ type $\mathrm{AB}$ [5]. The ABO system is determined by the presence of $A$ and $B$ antigens on the surface of RBCs and are the result of expression of three allelic genes $\mathrm{O}, \mathrm{A}$ and $\mathrm{B}$ on the chromosome no. 9 [6-8], the latter two being dominant to O. Depending on which blood group antigen is present on RBCs, the reciprocal antibody is found in plasma. When the antigen $A$ is found on RBCs, anti-B will be present in plasma; when the $B$ antigen is present on RBCs, anti-A will be found in the plasma. When there are no antigens present on RBCs as in blood 
group $\mathrm{O}$, both anti-A and anti-B antibodies will be found in plasma. Just the opposite is true for blood group $A B$.

Since both the ABO blood groups and malocclusion are genetic traits, it can be hypothesized that they have an association. In different countries, researches have been done to find the relationship between ABO blood groups and various systemic and oral conditions. Telen [9] listed well-known diseases such as ulcers, cancers of GIT, poliomyelitis, rheumatic fever, viral infections, bronchopneumonia and many other diseases which are related to and are affected by blood groups. Arneberg, et al. [10] studied the association of ABO blood groups with dental caries. He found less dental caries among secretors than among non-secretors of blood group substance. Ghamdi [11] studied the association between blood groups and severity of chronic periodontitis. Patients with blood group B appeared to be at greater risk of developing more severe forms of periodontitis. Koregol, et al. [12], stated that blood group type A showed a significantly higher percentage in the gingivitis group, blood group $\mathrm{O}$ showed a higher incidence in the periodontal group. The blood group $\mathrm{AB}$ showed the least percentage of periodontal diseases.

Few studies have also been conducted to determine the prevalence of different malocclusions in males and females. A study conducted on high school children in Italy showed the prevalence of Class II malocclusion in males over females. There was a higher incidence of increased overbite observed in males of central Anatolia. A study conducted in Japan showed a higher incidence of anterior cross-bite and crowding in girls $(1.56 \times)$ than boys. However, there was no statistically significant difference between males and females of Benin City, Nigeria.

Differences in malocclusion characteristics have been studied between different populations in previous studies e.g., Class III dental malocclusion is found to be common in Chinese, Japanese and Malaysian populations. Caucasians have been found to have Class II dental malocclusions in common. Class II dental malocclusion is found to be prevalent in Pakistan.

In general, many studies have been conducted to find out the association between blood groups and oral and dental pathologies, for instance, Sharma, et al. [13], some have succeeded; others have not due to the geographic diversity. Due to the lack of research on gender wise relationship between classes of malocclusion and $\mathrm{ABO}$ blood groups, this study was conducted. The purpose of this study was to find out the relationship of $\mathrm{ABO}$ blood groups with classes of malocclusion and relative prevalence of these characters in males and females of a defined population

\section{Research objectives}

The main objective of this study is to ascertain the association between blood group types and classes of dental malocclusion. The study has focused on the gender wise prevalence of classes of dental malocclusion.

\section{Hypotheses}

$\mathrm{H} 1$ : Is there any association between $\mathrm{ABO}$ blood groups and classes of dental malocclusion?

$\mathrm{H} 2$ : Is there any difference in the association of blood group type and classes of malocclusion in relation to gender wise prevalence?

\section{Materials and Methods}

\section{Research design}

The research design for this quantitative study was a cross-sectional research design.

\section{Sample size}

The current study was performed on 500 individuals with an age range of 15 to 45 years, along with permanent dentition only, reporting to the department of orthodontics of Punjab dental hospital, Lahore. Complete details about the classes of malocclusion and blood group of the subjects were noted in accordance with the $\mathrm{ABO}$ blood group system. The sample was divided into three groups. First was Class I group which served as a control group had 200 subjects were involved (74 males; 126 females), second was Class II group had 175 subjects were included ( 67 males; 108 females) and the third class was Class III group had 125 subjects were included ( 77 males; 48 females).

\section{Inclusion criteria}

Individuals with fully erupted permanent teeth in each arch excluding third molars were the participants of interest for this novel study. In the control group, only those individuals were included, which on examination manifested bilateral Angles Class I molar relationship with acceptable overjet and overbite (normal range 2-4 $\mathrm{mm}$ ). Furthermore, In Class II group, only those individuals were included who showed bilateral Angles Class II molar relationship while in Class III group, only those individuals were included who showed bilateral Angles Class III molar relationship.

\section{Exclusion criteria}

Patients with any systemic disease e.g., acute or chronic inflammatory or autoimmune diseases were not included in the sample. Those who presented with a history of trauma to the primary or permanent dentition or previous orthodontic treatment and with a history of smoking or steroidal and non-steroidal anti-inflammatory drugs were excluded cases. Moreover, the patients with oral mucosal lesions, periodontal diseases, root resorption, periapical lesions, or active caries and missing molars or retained deciduous teeth were not considered appropriate to fulfill the criteria of the present study.

\section{Apparatus}

- Sterile blood lancet or needle

- Sterile cotton or gauze swabs

- Clean and dry microscope slides

- Glass dropper with a long nozzle

- $1 \%$ sodium citrate in normal saline

- A microscope

\section{Materials}

\section{Reagents}

- Anti-A sera (blue): human polyclonal or murine monoclonal

- Anti-B sera (yellow): human polyclonal or murine monoclonal

- Anti-D sera (colorless) 


\section{Procedure}

After obtaining the consent from each subject, a separate sheet or Performa was used as a record for each individual including code given to the subject, age, gender, blood group and class of malocclusion. Evaluation of occlusal relationships was done at centric occlusion by asking the subject to swallow and then to occlude his/her teeth. Cheeks were fully retracted using tongue depressors to allow maximum visualization. Antero-posterior relationships of maxillary and mandibular first molars, in maximum intercuspation, were evaluated by Angle's classification.

Three large drops of blood were obtained from each subject's finger by piercing the skin with sterile lancet under aseptic conditions. The blood was placed on a clean and dry slide by "milking" or squeezing the finger. One drop of Anti-A sera was added to the first drop of blood. One drop of anti-B sera was added to the second drop and one drop of anti-D sera was added to the third drop of blood. Each was mixed using a clean toothpick and the slide was tilted back and forth. The slide was examined for agglutination of RBCs, first with the naked eye to see whether clumping has taken place (it appears as a coarse separation of RBCs in isolated clumps) then under low magnification microscope.

\section{Data analysis}

Data were analyzed statistically by using SPSS 21.0. Pearson Chisquare was used as statistical analysis to get the association of classes of malocclusion with blood group type.

\section{Result}

The gender wise statistical result of all the participants with classes of malocclusion i.e., Class I, Class II and Class III in relation to their respective blood groups is mentioned in Table 1 .

\begin{tabular}{|c|c|c|c|c|c|}
\hline \multirow[b]{2}{*}{ Gender } & & & \multicolumn{3}{|c|}{ Dental Malocclusion\% } \\
\hline & & & Class I & Class II & Class III \\
\hline \multirow[b]{4}{*}{ Male $(n=218)$} & \multirow[b]{4}{*}{ Blood group } & A & $18.90 \%$ & $13.40 \%$ & $9.10 \%$ \\
\hline & & B & $35.10 \%$ & $28.40 \%$ & $22.10 \%$ \\
\hline & & $A B$ & $13.50 \%$ & $41.80 \%$ & $44.20 \%$ \\
\hline & & 0 & $32.40 \%$ & $16.40 \%$ & $24.70 \%$ \\
\hline \multirow[b]{4}{*}{ Female $(n=282)$} & \multirow[b]{4}{*}{ Blood group } & A & $30.20 \%$ & $13.90 \%$ & $10.40 \%$ \\
\hline & & B & $27.80 \%$ & $40.70 \%$ & $43.80 \%$ \\
\hline & & $A B$ & $15.90 \%$ & $13.90 \%$ & $8.30 \%$ \\
\hline & & 0 & $26.20 \%$ & $31.50 \%$ & $37.50 \%$ \\
\hline
\end{tabular}

Chi-square, for males $=21.53, p=0.001, d f=6$; for females $=17.26, p=0.008, d f=6$

Table 1: Gender wise statistics of the sample in the three classes of malocclusion in relation to the blood groups.

The result of chi-square analysis revealed that there is a highly significant relationship between the types of blood groups and types of classes of malocclusion among both male $\left(\mathrm{r}^{2}=21.53, \mathrm{p}=0.001\right)$ and female gender $\left(r^{2}=17.26, p=0.008\right)$.
Furthermore, blood group type A was found to be associated with Class I malocclusion both in males (18.9\%) and females (30.2\%) as compared to the other two classes of malocclusion. But females with blood group A were found to be more in Class I of malocclusion than males.

For the blood group type B, the result revealed that among males, Class I of malocclusion was found to be highly associated (35.1\%) but among females, Class III of malocclusion was found to be greater (43.8\%). This means that males with blood group B were higher in Class I of malocclusion whereas females were found to be greater in number in Class III of malocclusion. While analyzing the third blood group category which was $\mathrm{AB}$ blood group, the result showed that Class III was found as a most prevalent type of malocclusion in males (44.2\%) whereas Class I was found to be the most prevalent one in females (15.9\%). From these finding, it can be assumed that males with $\mathrm{AB}$ blood group have a higher rate of Class III of malocclusion as compared to the females.

Lastly, the result of blood group $\mathrm{O}$ showed that males were greater in number in Class I of malocclusion (32.4\%) whereas females were more in Class III of malocclusion (37.5\%) as compared to the other two classes of malocclusion.

The Graph showed in Figure 1 depicts that highest prevalence in males found in blood group $\mathrm{AB}$ is of dental malocclusion Class III whereas in females, the highest prevalence of dental malocclusion was Class III but with the blood group type B. However, the lowest prevalent type of dental malocclusion was Class III with blood group type $\mathrm{A}$ in males and in females, the least prevalent type of dental malocclusion was Class III with blood group type AB. Other two classes of dental malocclusion, i.e., Class I and Class II, in all blood group types among both genders prevail in the medium frequency.

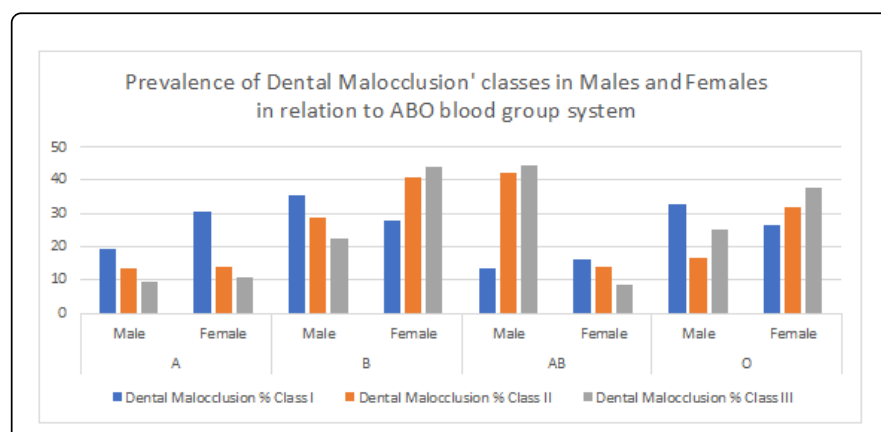

Figure 1: Prevalence of dental malocclusion' classes in males and females in relation to $\mathrm{ABO}$ blood group system.

\section{Discussion}

This novel research study was conducted to discover the prevalence of gender wise classes of dental malocclusion in relation to blood group type in the Pakistani context. Both the hypotheses of this study showed significant differences along with relationship existing among classes of malocclusion, blood group type, and gender-wise comparison. In Pakistani context, there is a highly significant relationship between the types of blood groups and types of classes of malocclusion among both male and female gender which was earlier concluded by Sharma, et al. [13], while finding an association between the blood group and dental malocclusion. 
Firstly, a higher level of association of blood group type A with Class I malocclusion both in males and females was found as compared to the other two classes of malocclusion. However, the prevalence of female gender with blood group A was higher in Class I of malocclusion than males.

Secondly, findings of the blood group type B revealed that among males, Class I of malocclusion was found to be highly associated whereas the prevalence of Class III of malocclusion is higher in females which showed the difference of both groups of gender. Thirdly, a strong association was observed between blood group type $A B$ and Class III of malocclusion in males, however, in females, the association was recorded between $\mathrm{AB}$ blood group type and Class $\mathrm{I}$ of malocclusion.

Lastly, while analyzing the relationship of classes of malocclusion and the blood group type $\mathrm{O}$ in both genders, the outcomes revealed the association to be more in males with Class I malocclusion, however, in females, the prevalence was higher in Class III of malocclusion as compared to the other two classes of malocclusion.

\section{Conclusion}

In conclusion, there was gender wise association difference between the classes of dental malocclusion and blood group types. The study also covered the gender wise prevalence of dental malocclusion in all the four blood groups which are $\mathrm{A}, \mathrm{B}, \mathrm{AB}$ and $\mathrm{O}$. The new findings will be helpful for clinicians with regards to preventive orthodontics. The research findings are a good and informative addition to the present literature as well as provides a ground for further research studies in the same area.

\section{Conflict of Interest Statement}

None to declare.

\section{References}

1. Daskalogiannakis J (ed.) (2000) Glossary of Orthodontic Terms. Quintessence Books.

2. Mossey PA (1999) The heritability of malocclusion: part 2. The influence of genetics in malocclusion. Br J Orthod 26: 195-203.

3. Dobzhansky T (1958) Evolution at work. Science 127: 1091-1096.

4. Landsteiner K (1901) Über Agglutinationserscheinungen normalen menschlichen blutes. Wien Klin Wochenschr 14: 1132-1134.

5. Behra D, Joshi D (2013) Distribution of ABO blood group and RH(D) factor in Western Rajasthan. J Med Res 3: 73-75.

6. Lewis M, Kaita H, Giblett ER, Anderson JE (1978) Genetic linkage analyses of chromosome 9 loci ABO and AK1. Cytogenet Cell Genet 22: 452-455.

7. Greenwalt TJ (1997) A short history of transfusion medicine. Transfusion 37: 550-563.

8. Telen MJ (1996) Erythrocyte blood group antigens: Polymorphisms of functionally important molecules. Semin Hematol 33: 302-314.

9. Muschel LH (1966) Blood groups, disease and selection. Bacteriol Rev 30: 427-441.

10. Arneberg P, Kornstad L, Nordbo H, Gjermo P (1976) Less dental caries among secretors than among non-secretors of blood group substance. Scand J Dent Res 84: 362-366.

11. Al Ghamdi AST (2009) Association between ABO blood groups and severity of chronic periodontitis. Medical Science 16: 31-41.

12. Koregol AC, Raghavendra M, Nainegali S, Kalburgi N, Varma S (2010) $\mathrm{ABO}$ blood groups and Rhesus factor: an exploring link to periodontal diseases. Indian J Dent Res 21: 364-368.

13. Sharma R (2015) Association of ABO blood groups with malocclusion in population of Jaipur, India: a prospective study. International Journal of Scientific Study 2: 45-51. 